\title{
Each to their own beat: periodicity in temporal inference
}

\author{
Asma Motiwala*, Charles Fox, Tony Prescott \\ From Twentieth Annual Computational Neuroscience Meeting: CNS*2011 \\ Stockholm, Sweden. 23-28 July 2011
}

Characterizing cortical computation has been a key challenge in neuroscience. It has been suggested that the cerebral cortex is involved in unsupervised learning [1] and the notion that cortical computation is probabilistic is being increasingly accepted [2]. It has also been shown that cortical regions are hierarchically connected [3] and this has important implications on the way information could be represented. Moreover, the classical notion that processing in the cortex is predominantly governed by its feedforward inputs has been largely replaced by the view that feedback signals and other contextual information play a crucial role in the way information is encoded and learnt $[2,4,5]$. There is increasing emphasis on the influence of ongoing activity [5], ie. the state of the network on the effects of feedforward inputs.

The hierarchical temporal memory (HTM) model of cortical computation [6] was used as the starting point for the current work. HTM is a hierarchical probabilistic model that uses spatial and temporal characteristics of its inputs to represent 'invariant causes' in the world. The key assumptions in the model are that learning in the cortex involves learning sequences of coincident patterns in its inputs and that the temporal patterns in inputs are critical for the brain's ability to learn and infer with ambiguous sensory information.

For the current work we are investigating tactile perception and implementing the HTM model using sensory inputs from artificial whiskers. The HTM model emphasizes that temporal patterns in inputs are extremely important features for learning and inference in the brain. This is especially the case with tactile perception since very little information is available at any single instant; how sensory inputs evolve over time conveys indispensable information about stimuli in the world.

\footnotetext{
* Correspondence: a.motiwala@sheffield.ac.uk

Department of Psychology, University of Sheffield, Sheffield, S10 2TN, UK

Full list of author information is available at the end of the article
}

In modeling unsupervised learning, 'time' is commonly treated as indexing sequential inputs [7]. However, we are suggesting that learning, inference and prediction with temporal patterns pose a theoretical need to model some mechanism to encode time not only in terms of a temporal succession of events and causes, but also as a metric of periodicity. This theoretical need is perfectly aligned with well established empirical evidence that the brain engages in a plethora of oscillations at several different scales and frequencies. Our implementation of HTM incorporates a mechanism which attempts to mimic hypothesized effects of local neural oscillations in temporal inference.

We are suggesting that cortical networks can dynamically maintain local oscillations to best match the relative frequencies with which inputs tend to occur. Assuming that all inputs to any cortical network are superimposed on its ongoing activity, these local oscillations can discretize the continuous stream of sensory inputs in signaling to subsequent levels in the hierarchy, as well as modulate the signal to suppress or enhance effects of specific components in the inputs based on their timing. We are demonstrating these effects in our implementation of HTM.

Published: 18 July 2011

\section{References}

1. Doya K: What are the computations of the cerebellum, the basal ganglia and the cerebral cortex? Neural networks 1999, 12:961-974.

2. Knill DC, Pouget A: The Bayesian brain: the role of uncertainty in neural coding and computation. TRENDS in Neurosciences 2004, 12:712-719.

3. Felleman DJ, Van Essen DC: Distributed hierarchical processing in the primate cerebral cortex. Cerebral cortex 1991, 1:1-47.

4. Thomson AM, Bannister AP: Interlaminar connections in the neocortex. Cerebral Cortex 2003, 13:5-14.

5. Arieli A, Sterkin A, Grinvald A, Aertsen A: Dynamics of ongoing activity: explanation of the large variability in evoked cortical responses. Science 1996, 273:1868-1871.
C Biomed Central

() 2011 Motiwala et al; licensee BioMed Central Ltd. This is an open access article distributed under the terms of the Creative Commons Attribution License (http://creativecommons.org/licenses/by/2.0), which permits unrestricted use, distribution, and reproduction in any medium, provided the original work is properly cited. 
6. George D, Hawkins J: Towards a mathematical theory of cortical microcircuits. PLoS Comput Biol 2009, 5:e1000532.

7. Bishop CM: Pattern recognition and machine learning. NewYork: Springer; 2006.

doi:10.1186/1471-2202-12-S1-P257

Cite this article as: Motiwala et al:: Each to their own beat: periodicity in temporal inference. BMC Neuroscience 2011 12(Suppl 1):P257.

Submit your next manuscript to BioMed Central and take full advantage of:

- Convenient online submission

- Thorough peer review

- No space constraints or color figure charges

- Immediate publication on acceptance

- Inclusion in PubMed, CAS, Scopus and Google Scholar

- Research which is freely available for redistribution

Submit your manuscript at www.biomedcentral.com/submit 